\title{
Rainbow Fourier transform
}

\author{
Mikhail D. Alexandrov $^{\mathrm{a}, \mathrm{b}, *}$, Brian Cairns ${ }^{\mathrm{b}}$, Michael I. Mishchenko ${ }^{\mathrm{b}}$ \\ a Department of Applied Physics and Applied Mathematics, Columbia University, 2880 Broadway, New York, NY 10025, USA \\ ${ }^{\mathrm{b}}$ NASA Goddard Institute for Space Studies, 2880 Broadway, New York, NY 10025, USA
}

\section{A R T I C L E I N F O}

Available online 30 March 2012

\section{Keywords:}

Electromagnetic scattering

Polarization

Mie theory

Rainbow

Optical particle characterization

Remote sensing

\begin{abstract}
A B S T R A C T
We present a novel technique for remote sensing of cloud droplet size distributions. Polarized reflectances in the scattering angle range between $135^{\circ}$ and $165^{\circ}$ exhibit a sharply defined rainbow structure, the shape of which is determined mostly by single scattering properties of cloud particles, and therefore, can be modeled using the Mie theory. Fitting the observed rainbow with such a model (computed for a parameterized family of particle size distributions) has been used for cloud droplet size retrievals. We discovered that the relationship between the rainbow structures and the corresponding particle size distributions is deeper than it had been commonly understood. In fact, the Mie theory-derived polarized reflectance as a function of reduced scattering angle (in the rainbow angular range) and the (monodisperse) particle radius appears to be a proxy to a kernel of an integral transform (similar to the sine Fourier transform on the positive semi-axis). This approach, called the rainbow Fourier transform (RFT), allows us to accurately retrieve the shape of the droplet size distribution by the application of the corresponding inverse transform to the observed polarized rainbow. While the basis functions of the proxy-transform are not exactly orthogonal in the finite angular range, this procedure needs to be complemented by a simple regression technique, which removes the retrieval artifacts. This non-parametric approach does not require any $a$ priori knowledge of the droplet size distribution functional shape and is computationally fast (no look-up tables, no fitting, computations are the same as for the forward modeling).
\end{abstract}

(c) 2012 Elsevier Ltd. All rights reserved.

\section{Introduction}

Cloud droplet size retrievals from measurements in the solar spectral domain can be performed using both total and polarized reflectances, defined respectively as

$R=\frac{\pi I}{\mu_{s} I_{0}} \quad$ and $\quad R_{p}=-\frac{\pi Q}{\mu_{s} I_{0}}$,

where $I_{0}$ is the extraterrestrial solar irradiance and $\mu_{s}$ is the cosine of the solar zenith angle (SZA). The Stokes

\footnotetext{
* Corresponding author at: Department of Applied Physics and Applied Mathematics, Columbia University, 2880 Broadway, New York, NY 10025, USA. Tel.: +1 212678 5548; fax: +1212678 5552 .

E-mail address: mda14@columbia.edu (M.D. Alexandrov).
}

parameter $Q$ in Eq. (1) is defined with respect to the scattering plane containing both solar and view directions. In the case of water cloud, the parameter $U$ in this plane is 2-3 orders of magnitude smaller than $Q$ (cf. [1]), and can be neglected. This allows us to use Eq. (1) as the definition of polarized reflectance based on the signed degree of linear polarization (cf. [2]). Retrievals of the cloud droplet size from polarized reflectance measurements in the rainbow angular range between $135^{\circ}$ and $165^{\circ}[3,4,1]$ are almost free of uncertainties due to the 3D nature of radiation and to gaseous and aerosol absorptions, which affect the remote sensing methods based solely on the multispectral measurements (not including polarization, cf. $[5,6])$. This advantage is due to the fact that the rainbow shape (Fig. 1) is dominated by single 

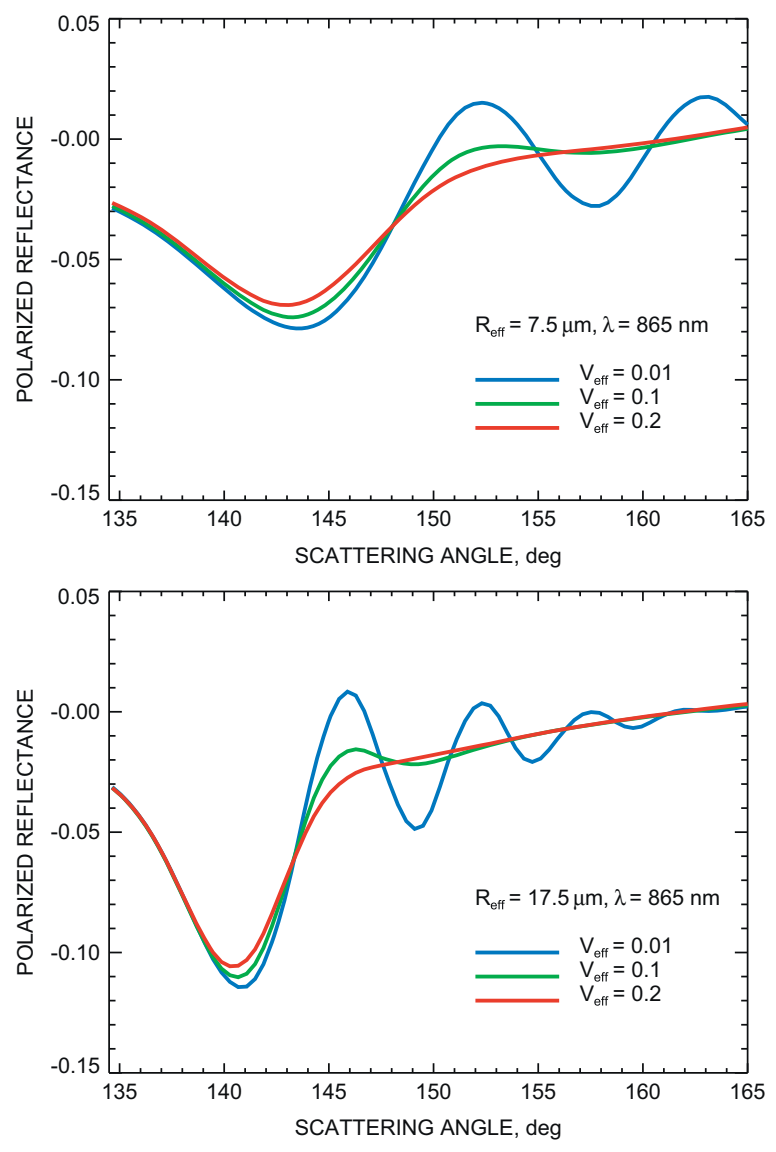

Fig. 1. Examples of polarized reflectances in the rainbow scattering angle range. These radiative transfer simulations were made for realistic cloud droplet size distributions with $r_{\text {eff }}=7.5 \mu \mathrm{m}$ (top) and $17.5 \mu \mathrm{m}$ (bottom). In both cases the gamma distribution model was used with $v_{\text {eff }}=0.01,0.1$, and 0.2 , and the cloud optical depth was assumed to be 5 .

scattering of light by cloud particles. To account for the small contributions from all other factors (multiple scattering, Rayleigh scattering, aerosol extinction, ground surface reflectance for thin clouds, as well as effects caused by rotation to the scattering plane in Eq. (1)), the measured polarized reflectance was fit by the expression of the form

$R_{p}(\theta)=A \cdot P_{12}^{(\text {Mie })}[\theta ; n(r)]+B \cdot \theta+C$,

where $\theta$ is the scattering angle, $n(r)$ is the cloud droplet size distribution parameterized by its effective radius $r_{\text {eff }}$ and variance $v_{\text {eff }}$ (the gamma distribution shape is assumed), while $A, B$, and $C$ are the empirical fitting parameters (the linear term in $\theta$ can be replaced with a Rayleigh-like term $\sim \cos ^{2} \theta$ [1]). The phase matrix elements $P_{12}^{(\text {Mie })}$ are computed using the Mie theory for a grid of $r_{\text {eff }}$ and $v_{\text {eff }}$ values. Alexandrov et al. [1] demonstrated that this retrieval approach, while being very accurate, has a disadvantage associated with the necessity of selecting an a priori functional shape of the droplet size distribution. If this shape is assumed to be monomodal, while the retrieval algorithm is applied to the data from a cloud with a bimodal droplet distribution (e.g., including drizzle droplets), the retrieved effective size will be biased toward that of the dominant mode (this is a common feature of all least square fit retrievals).

In this study we present an alternative retrieval approach based on our observation that the polarized reflectances in the rainbow region taken as functions of the (reduced) scattering angle, while parameterized by particle size, form a basis for an approximate integral transform with certain similarities to the sine Fourier transform on the positive semi-axis. This transformation, which we called the rainbow Fourier transform (RFT), turned out to have a simple inverse (as the ordinary Fourier transform). While the direct RFT acting from the space of droplet size distributions (functions of the particle radius $r$ ) into the space of rainbow polarized reflectances (functions of the scattering angle $\theta$ ) is a simple integration used in direct modeling, its inverse, acting in the opposite direction, is a powerful retrieval tool for remote sensing of cloud microphysical properties.

We will describe the difference between the exact and "approximate" transforms and show how to construct the accurate inverse RFT on examples of droplet size distributions of various shapes (such as rectangular and bimodal gamma distribution). We will also present some analytical expressions derived from approximations to the Mie theory, which indirectly support our method. However, we currently have no theoretical proof of the RFT existence based on the Mie theory, and our method is justified by empirical choices and is validated by numerical experiments.

In practice, the proposed retrieval method is intended to be used for analyses of measurements made by the Research Scanning Polarimeter (RSP) [7-13]. This instrument is an airborne prototype for the satellite Aerosol Polarimetery Sensor (APS), which was built as part of the NASA Glory Mission [14]. The RSP measures the I, $Q$ and $U$ components of the Stokes vector in nine spectral channels with center wavelengths of $410,470,555,670$, $865,960,1590,1880$ and $2250 \mathrm{~nm}$. It is a push-broom sensor scanning along the aircraft track within $\pm 60^{\circ}$ from nadir (starting at forward direction) and making samples at $0.8^{\circ}$ intervals. Thus, each scan consists of about 150 instantaneous measurements. The data from the actual RSP scans is then aggregated into "virtual" scans, each consisting of all reflectances (at a variety of scattering angles) from a single point on the ground or at the cloud top. In recent years the RSP has been deployed onboard different aircrafts during a number of field campaigns, making measurements for a vast variety of cloud scenes.

\section{Computation of the phase matrix element $\boldsymbol{P}_{12}$ for a polydisperse cloud}

The averaged phase matrix element $\bar{P}_{12}(\theta)$ for a droplet size distribution $n(r)$, normalized by the condition

$\int_{0}^{\infty} n(r) \mathrm{d} r=1$ 
is given by the expression

$\bar{P}_{12}(\theta)=\frac{\int_{0}^{\infty} \sigma_{s c a}(r) P_{12}(r, \theta) n(r) \mathrm{d} r}{\int_{0}^{\infty} \sigma_{s c a}(r) n(r) \mathrm{d} r}$.

Here, we use the high-resolution functions $P_{12}(r, \theta)$ and the scattering cross-section $\sigma_{s c a}(r)$ computed according to the Mie theory for the given wavelength. This dataset was initially developed for generating look-up tables for RSP/APS retrievals and has an angular resolution of $0.2^{\circ}$. It was produced by averaging the Mie theory results over a set of very narrow size distributions (triangular, of width $0.1 \mu \mathrm{m}$ spaced every $0.05 \mu \mathrm{m}$ up to the maximum radius of $100 \mu \mathrm{m}$ ). For our purposes, we can neglect the finite width of these distributions and consider the dataset as a non-averaged Mie theory output. In this study we use Mie datasets for three wavelengths representing blue, red, and IR parts of the spectrum: $410.2,863.5$, and $2265.1 \mathrm{~nm}$. The respective complex refractive indices of water used in these computations are given in Table 1.

Eq. (4) can be written as

$\bar{P}_{12}(\theta)=\int_{0}^{\infty} P_{12}(r, \theta) n_{\sigma}(r) \mathrm{d} r$,

where $n_{\sigma}(r)$ is the "scattering" distribution

$n_{\sigma}(r)=\frac{\sigma_{s c a}(r) n(r)}{\int_{0}^{\infty} \sigma_{s c a}(r) n(r) \mathrm{d} r}$

It is normalized by the same condition (3) as $n(r)$. The scattering cross-section $\sigma_{\text {sca }}$ can be expressed as

$\sigma_{s c a}(r)=\pi r^{2} Q_{s c a}(r)$,

where $Q_{s c a}(r)$ is the scattering efficiency. In the case of large and practically non-absorbing cloud particles $Q_{s c a}=Q_{e x t} \approx 2$, thus, $n_{\sigma}(r)$ is close to the area distribution

$n_{a}(r)=\frac{r^{2} n(r)}{\int_{0}^{\infty} r^{2} n(r) \mathrm{d} r}$

normalized by

$\int_{0}^{\infty} n_{a}(r) \mathrm{d} r=1$.

Let us introduce the reduced scattering angle

$\gamma=\theta-\theta_{0}$

where $\theta_{0}$ is close to the rainbow angle $\theta_{R}$ [15], but actually is an adjustment parameter. The angle $\gamma$ varies from 0 to the angular width of the rainbow region

Table 1

Refractive indices and rainbow angles for the three wavelengths used in this study.

\begin{tabular}{llll}
\hline \multirow{4}{*}{ Wavelength $(\mathrm{nm})$} & \\
\cline { 2 - 4 } & 410.2 & 863.5 & 2265.1 \\
\hline$n_{r}$ & 1.3426514 & 1.3275359 & 1.2815182 \\
$n_{i}$ & $1.66 \times 10^{-9}$ & $3.49 \times 10^{-7}$ & $4.17 \times 10^{-4}$ \\
$\theta_{R}$ & $139.3^{\circ}$ & $137.1^{\circ}$ & $129.8^{\circ}$ \\
$\theta_{0}$ & $137.5^{\circ}$ & $134.5^{\circ}$ & $123.5^{\circ}$ \\
\hline
\end{tabular}

$\left(\approx 30^{\circ}\right)$. However, we can formally extend this range to infinity. Let us define the following functions of $\gamma$ :

$p(\gamma)=\bar{P}_{12}\left(\theta_{0}+\gamma\right)$

and

$F(r, \gamma)=P_{12}\left(r, \theta_{0}+\gamma\right)$.

In this notation

$p(\gamma)=\int_{0}^{\infty} F(r, \gamma) n_{a}(r) \mathrm{d} r$,

which can be interpreted as the result of an integral transform in the $(r, \gamma)$-space mapping the area distribution $n_{a}(r)$ to its image $\hat{n}_{a}(\gamma)=p(\gamma)$. The function $F(r, \gamma)$ is the kernel of this transform.

\section{Integral transforms on the positive semi-axis}

An integral transform in $(r, \gamma)$-space, where $r \geq 0$ and $\gamma \geq 0$, and its inverse have the respective general forms

$\hat{f}(\gamma)=\int_{0}^{\infty} f(r) F(r, \gamma) w_{r}(r) \mathrm{d} r$,

$f(r)=\int_{0}^{\infty} \hat{f}(\gamma) F(r, \gamma) w_{\gamma}(\gamma) \mathrm{d} \gamma$

where $w_{r}$ and $w_{\gamma}$ are the corresponding weighting functions. These expressions imply the orthogonality of the basic functions $F(r, \gamma)$ :

$\int_{0}^{\infty} F\left(r_{1}, \gamma\right) F\left(r_{2}, \gamma\right) w_{\gamma}(\gamma) \mathrm{d} \gamma=\frac{\delta\left(r_{1}-r_{2}\right)}{w_{r}\left(r_{1}\right)}$

and

$\int_{0}^{\infty} F\left(r, \gamma_{1}\right) F\left(r, \gamma_{2}\right) w_{r}(r) \mathrm{d} r=\frac{\delta\left(\gamma_{1}-\gamma_{2}\right)}{w_{\gamma}\left(\gamma_{1}\right)}$.

Let us consider a family of basic functions of the form $G=G(r \gamma)$, which are orthogonal with unit weight $w_{r}=$ $w_{\gamma}=1$ :

$\int_{0}^{\infty} G\left(r_{1} \gamma\right) G\left(r_{2} \gamma\right) \mathrm{d} \gamma=\delta\left(r_{1}-r_{2}\right)$

$\int_{0}^{\infty} G\left(r \gamma_{1}\right) G\left(r \gamma_{2}\right) \mathrm{d} r=\delta\left(\gamma_{1}-\gamma_{2}\right)$

An example of such a family is

$G(r \gamma)=\sqrt{\frac{2}{\pi}} \sin r \gamma$

which forms the basis of the sine Fourier transform (a similar example can be constructed by replacing sine with cosine in this expression). Other families of orthogonal basic functions can be created by replacing $r \rightarrow r^{\alpha}$ and $\gamma \rightarrow \gamma^{\beta}$ in the argument of $G$ :

$F(r, \gamma)=r^{v} \gamma^{\mu} G\left(r^{\alpha} \gamma^{\beta}\right)$

A straightforward substitution demonstrates that these functions obey Eqs. (14)-(17) with the weights

$w_{r}(r)=\alpha r^{\alpha-2 v-1} \quad$ and $\quad w_{\gamma}(\gamma)=\beta \gamma^{\beta-2 \mu-1}$. 


\section{Analytical approximations of $P_{12}$}

The amplitude scattering matrix (cf. [16]) relates the components of the incident electric field (propagating in the positive $z$-direction) to that of the scattered radiation:

$$
\left(\begin{array}{c}
E_{r}^{s} \\
E_{s}^{i}
\end{array}\right)=\frac{\exp (-i k R+i k z)}{i k R}\left(\begin{array}{cc}
S_{1} & S_{4} \\
S_{3} & S_{2}
\end{array}\right)\left(\begin{array}{c}
E_{r}^{i} \\
E_{l}^{i}
\end{array}\right)
$$

Here, $R$ is the distance from the particle (in the far-field), $k=2 \pi / \lambda$ is the wave number ( $\lambda$ is the light wavelength), the indices $r$ and $l$ represent the components of $\mathbf{E}$ in any two mutually orthogonal directions. In the case of spherical particles, the amplitude scattering matrix is diagonal: $S_{3}=S_{4}=0$. The Stokes vectors $\mathbf{I}=\{I, Q, U, V\}$ of the incident and scattered radiation are related by

$\mathbf{I}=\frac{\sigma_{s c a}}{4 \pi R^{2}} \mathbf{P} \mathbf{I}_{\mathbf{0}}$,

where $\mathbf{P}$ is the $4 \times 4$ phase matrix. The element $P_{12}$ determining polarized reflectance is expressed in terms of the amplitude scattering matrix elements as

$P_{12}=\frac{2 \pi}{k^{2} \sigma_{s c a}}\left(\left|S_{1}\right|^{2}-\left|S_{2}\right|^{2}\right)$.

An idea of the functional dependence of $P_{12}$ on the size parameter

$\beta=k r=\frac{2 \pi r}{\lambda} \quad$ or $\quad \kappa=2 \beta$

( $r$ is the spherical particle radius) and on the reduced scattering angle in the rainbow region $\epsilon=\theta-\theta_{R}$ (here $\theta_{R}$ is the wavelength-dependent rainbow angle, see Table 1) can be obtained from the Airy approximation [15]. This analytical approximation is formally valid for very large size parameters $\beta \gtrsim 5000$ and very small reduced angles $\epsilon \leq 0.5^{\circ}$; however, as we show below, it can lead to an approximate formula for $P_{12}$ which is accurate in a wider parameter range. The expression for the amplitude scattering matrix elements in the Airy approximation is

$S_{j} \approx-2 e^{i \varphi} \frac{\sqrt{\pi} n u_{j}}{\sqrt{\sin \theta}} \kappa^{7 / 6} \mathrm{Ai}\left(-0.369 \kappa^{2 / 3} \epsilon\right)$

$j=1,2$. Here, $n$ is the real part of the refractive index of water, $u_{1}=0.0381, u_{2}=0.00786$, and $e^{i \varphi}$ is a phase factor irrelevant for absolute value computation. Substituting Eq. (27) into Eq. (25) yields

$P_{12} \approx \frac{0.04 \pi}{\sin \theta} \kappa^{1 / 3} \mathrm{Ai}^{2}\left[-0.369 \kappa^{2 / 3} \epsilon\right]$.

The functions $\mathrm{Ai}^{2}(z)$ do not obey orthonormality relations suitable for our purposes (cf. [17]), so we need to use further approximations. We use the fact that the Airy function $\operatorname{Ai}(z)$ is close to its asymptotic approximation [18] for a large negative argument

$\operatorname{Ai}(-z) \approx \frac{z^{-1 / 4}}{\sqrt{\pi}} \sin \left(\frac{2}{3} z^{3 / 2}+\frac{\pi}{4}\right)$ even if the argument value is moderate. This leads to

$\operatorname{Ai}^{2}(-z) \approx \frac{z^{-1 / 2}}{2 \pi}\left[1+\sin \left(\frac{4}{3} z^{3 / 2}\right)\right]$

and to another approximate formula for $P_{12}$ :

$P_{12} \approx \frac{0.033}{\sin \theta \epsilon^{1 / 2}}\left[1+\sin \left(0.3 \kappa \epsilon^{3 / 2}\right)\right]$.

As mentioned above, the Airy approximation (28) is formally valid only in the very close vicinity of $\theta_{R}$ : $\epsilon \ll \kappa^{-1 / 3} \ll 1$, that is a few degrees. While Fig. 2 (top) demonstrates precisely this, it also shows that the exact Mie function and the Airy approximation exhibit certain similarity within the entire rainbow range $\left(135^{\circ}-165^{\circ}\right)$. It is also seen in this plot that $\mathrm{Ai}^{2}(x)$ in Eq. (28) is very closely approximated by its large-argument asymptotics leading to Eq. (31). The exception is only for its first quarter-period, while some deviations at substantially larger arguments simply indicate the need for a more precise asymptotic formula [18]. Based on Eq. (31) the following analytical approximation for $P_{12}$ can be introduced:

$P_{12} \approx-\frac{0.035}{\epsilon^{3 / 4}} \sin \left(0.358 \kappa \epsilon^{1.605}\right)+0.1 \epsilon-0.05$.

Its plot is shown in comparison with the exact curve in Fig. 2 (bottom). Note that we are not looking for a high precision approximation here, but only for an adequate analytical expression to be used for educated guess of the integral transform weights.

\section{Definition of RFT}

We can take the functional form of the first term in Eq. (32) as a proxy for the integral transform kernel (12):

$F(r, \gamma) \sim \gamma^{-3 / 4} \sin \left(r \gamma^{3 / 2}\right)$

Here, $\gamma$ replaces $\epsilon$, giving us the freedom to choose $\theta_{0}$ different from $\theta_{R}$, and the particle radius $r$ replaces the size parameter $\kappa$. We omit all constant factors for clarity and use $1.605 \approx 3 / 2$ (which makes a rather small phase change in Eq. (32)). This expression coincides with Eq (21), where the function $G(x) \propto \sin x$ (Eq. (20)), and

$\alpha=1, \quad \beta=\frac{3}{2}, \quad v=0, \quad \mu=-\frac{3}{4}$.

Thus, according to Eq. (22) the corresponding weighting functions are

$w_{\gamma}=\gamma^{2}$ and $\quad w_{r}=1$.

This allows us to formally define the rainbow Fourier transform (RFT) of the area size distribution $n_{a}(r)$ as

$\hat{n}_{a}(\gamma)=\int_{0}^{\infty} n_{a}(r) F(r, \gamma) \mathrm{d} r$

and its inverse

$n_{a}^{\prime}(r)=\int_{0}^{\gamma_{\max }} \hat{n}_{a}(\gamma) F(r, \gamma) \gamma^{2} \mathrm{~d} \gamma$

where the integration is performed within the rainbow region $\left(\gamma_{\max } \approx 30^{\circ}\right)$, and we use the exact kernels $F(r, \gamma)$ derived from the Mie theory (Eq. (12)) rather than their approximations. It appears that these functions are not 

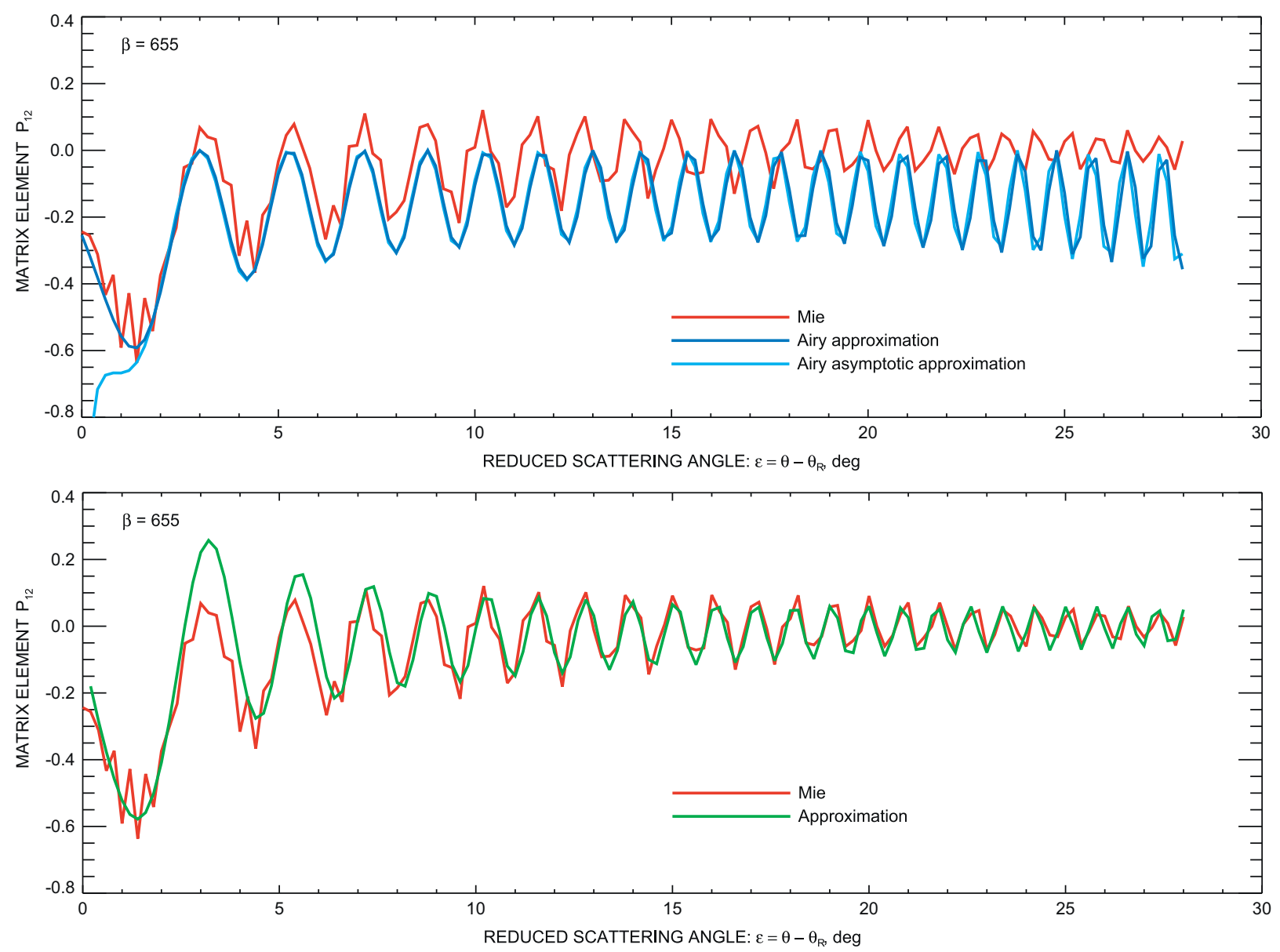

Fig. 2. Exact (derived from the Mie theory, red) and approximate dependences of the phase matrix element $P_{12}$ on the reduced scattering angle $\epsilon=\theta-\theta_{R}$. Top: comparison with the Airy approximation Eq. (28) (dark blue) and the asymptotic formula Eq. (31)) (light blue). Bottom: Comparison with the approximation of Eq. (32) (green). All computations were made for the size parameter $\beta=655$, a $863 \mathrm{~nm}$ wavelength, and $\theta_{R}=137^{\circ}$. (For interpretation of the references to color in this figure legend, the reader is referred to the web version of this article.)

precisely orthogonal, so the loop-transform (direct followed by inverse) is not an identity operator. This means that $n_{a}^{\prime} \neq n_{a}$, but rather

$n_{a}^{\prime}(r) \approx C_{1} \cdot n_{a}(r)+C_{2}+$ noise,

where $C_{1}$ and $C_{2}$ are constants, while "noise" stands for artifacts to be addressed in the next section.

The choice of the wavelength-dependent angle $\theta_{0}$ is made by keeping $n_{a}^{\prime}(r)$ constant, where $n_{a}(r)=0$. If $\theta_{0}$ is too small, the bottom-line of $n_{a}^{\prime}(r)$ tends to be convex (having maximum around $50 \mu \mathrm{m}$ ), while if $\theta_{0}$ is too high, the bottom-line is concave. The values of $\theta_{0}$ determined in this way are summarized in Table 1 with the corresponding rainbow angles shown for comparison. The values of $\theta_{0}$ appear to be $2-6^{\circ}$ lower than the corresponding angles $\theta_{R}$. Fig. 3 presents examples of RFT's base functions (12) vs. the reduced angle $\gamma$ for 410,863 , and $2265 \mathrm{~nm}$ wavelengths. These plots show that at $\theta=\theta_{0}(\gamma=0)$ the base functions are close to 0 , while $\theta_{R}$ is in the middle of their first half-period (as it is in the Airy approximation [15]). It is also clear from these plots that the curves corresponding to the 410 and $863 \mathrm{~nm}$ wavelengths have a more regular structure than that at $2265 \mathrm{~nm}$. This may be caused by stronger absorption of water at the latter wavelength (see Table 1). Unfortunately, this lack of structure impairs the retrievals significantly, hence we will present the results only for 410 and $863 \mathrm{~nm}$ wavelengths.

Fig. 4 shows the results of application of the looptransform to two area size distributions $n_{a}(r)$ of different functional shapes: a bimodal gamma distribution [16] and a rectangular distribution (which is constant over a selected interval, and zero otherwise). The value of the constant $C_{2}$ in Eq. (38) is determined from the largedroplet range $r \sim 90-100 \mu \mathrm{m}$, where $n_{a}(r)$ is assumed to be zero. This value is subtracted from the retrieved $n_{a}^{\prime}(r)$, after which the constant $C_{1}$ is supposed to be determined from normalization condition Eq. (9). However, for our tests, when the initial $n_{a}(r)$ is known, we simply scale the returned distribution so it has the same maximum value as the initial one (in the case of the rectangular distribution the median over the distribution top is taken instead of the absolute maximum). The initial distributions $n_{a}(r)$ are over-plotted (in black) for comparison. We use the 

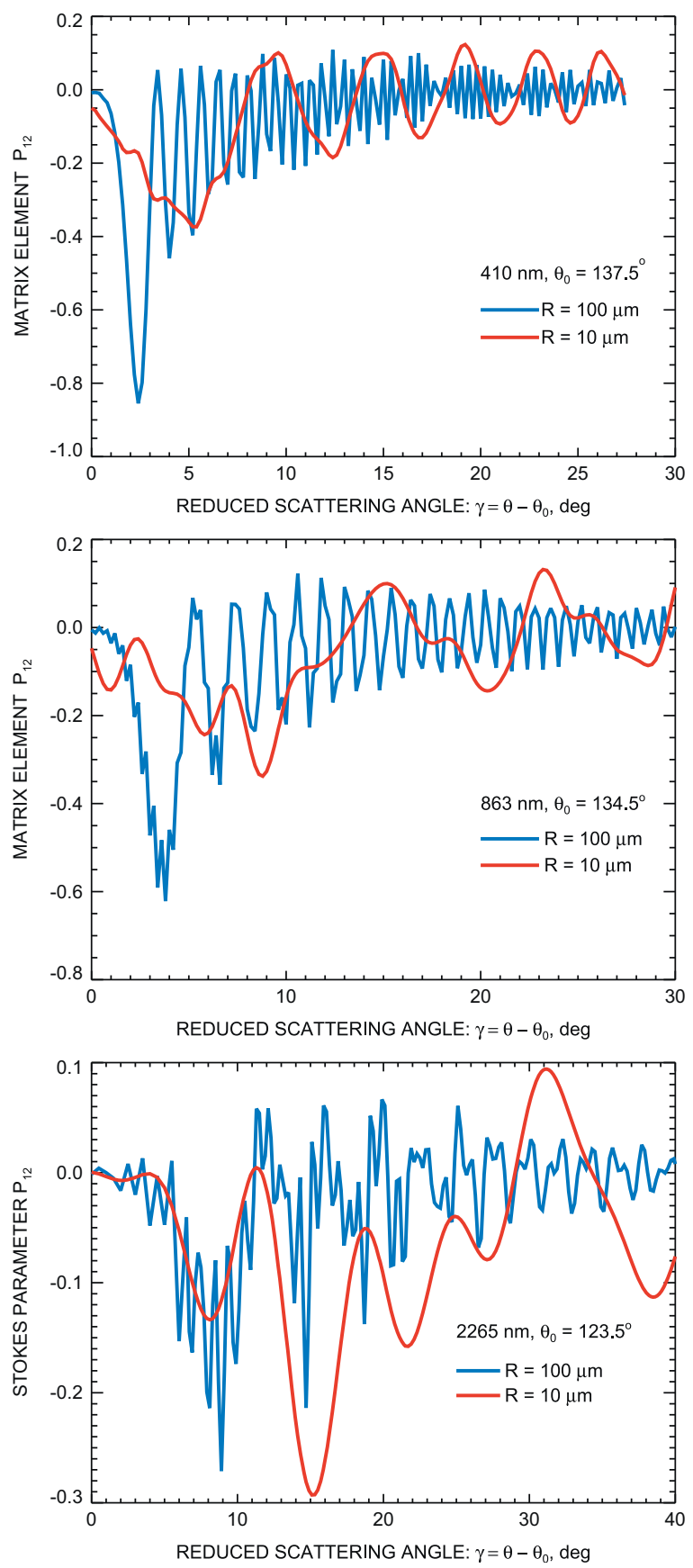

Fig. 3. Examples of RFT's basis functions $F(r, \gamma)$ vs. the reduced angle $\gamma$ for 10 and $100 \mu \mathrm{m}$ droplet radii and different wavelengths: $410 \mathrm{~nm}$ (top), $863 \mathrm{~nm}$ (middle), and $2265 \mathrm{~nm}$ (bottom).

metric [19]

$\Delta=\frac{1}{2} \int_{0}^{r_{\max }}\left|n_{a}^{\prime \prime}(r)-n_{a}(r)\right| \mathrm{d} r$

to quantify how close the retrieved distribution is to the initial one. Here $n_{a}^{\prime \prime}(r)$ denotes the loop-RFT result of Eq. (38) after the correction for the constants $C_{1}$ and $C_{2}$ described above. If both distributions $n_{a}^{\prime \prime}(r)$ and $n_{a}(r)$ are normalized to unity according to Eq. (9), this metric represents the fraction of the droplet ensemble that is "misplaced" in the retrieved $n_{a}^{\prime \prime}(r)$ relative to the initial distribution $n_{a}(r) . \Delta=0$ would indicate that $n_{a}^{\prime \prime}(r) \equiv n_{a}(r)$, while $\Delta=1$ corresponds to the case when $n_{a}^{\prime \prime}(r)$ and $n_{a}(r)$ do not have common support. In practice, normalization of $n_{a}^{\prime \prime}(r)$ can be affected by retrieval errors, causing $\Delta$ to exceed 1. However, we still regard this metric as an adequate measure of the retrieval accuracy, which is conveniently expressed in per cent. We consider the accuracy to be good if $\Delta \lesssim 10 \%$.

\section{Correction for weak orthogonality artifacts}

While the loop-transform results presented in Fig. 4 show generally good resemblance to the initial size distributions, some significant artifacts still contaminate the retrievals causing large values of $\Delta \sim 30 \%$. We assume that these artifacts are caused mainly by the imperfect orthogonality of the RFT basis functions $F(r, \gamma)$. We modeled the effects of the weak orthogonality analytically (see Appendix A) assuming that our basis functions can be represented in the form

$F(r, \gamma)=c H(r, \gamma)+g(r, \gamma)$,

where $c$ is a constant, $H(r, \gamma)$ do satisfy appropriate orthogonality conditions, while $g(r, \gamma)$ is an additional term depending mainly on $\gamma$ and only weakly on $r$ (note that we do not know anything more specific about these components). Computations presented in Appendix $A$ show that these assumptions lead to the expression for the loop-transform result similar to Eq. (38)

$n_{a}^{\prime}(r)=c^{2} n_{a}(r)+\eta(r)$,

where the correction function $\eta$ responsible for "noise" can be represented as

$\eta(r)=\eta_{u}(r)+\eta_{s}(r)+\eta_{g}(r)+C_{\eta}$.

Here, $\eta_{u}(r)$ is a universal (independent of the size distribution $\left.n_{a}(r)\right)$ correction function, $C_{\eta}$ is a (distribution-dependent) constant, while $\eta_{s}(r)$ and $\eta_{g}(r)$ are distribution-dependent corrections, which, however, are substantially smaller in magnitude than $\eta_{u}(r)$. The function $\eta_{s}(r)$ has a rapidly oscillating structure and can be represented in the form

$\eta_{s}(r)=B_{1} s_{1}(r)+B_{0} s_{0}(r)$,

where $B_{0}$ and $B_{1}$ are (distribution-dependent) constants, while

$s_{0}(r)=\int_{0}^{\gamma_{\max }} F(r, \gamma) \gamma^{2} \mathrm{~d} \gamma$

and

$s_{1}(r)=\int_{0}^{\gamma_{\max }} \gamma F(r, \gamma) \gamma^{2} \mathrm{~d} \gamma$

are universal functions. The function $\eta_{g}(r)$ is assumed to be slowly varying, while its specific structure is unknown.

Based on this model consideration and results of numerical tests, we can design a correction procedure allowing the separation and removal of the artifacts from $n_{a}^{\prime}(r)$, and the restoration of the original distribution $n_{a}(r)$. We should note that, as mentioned above, the total 

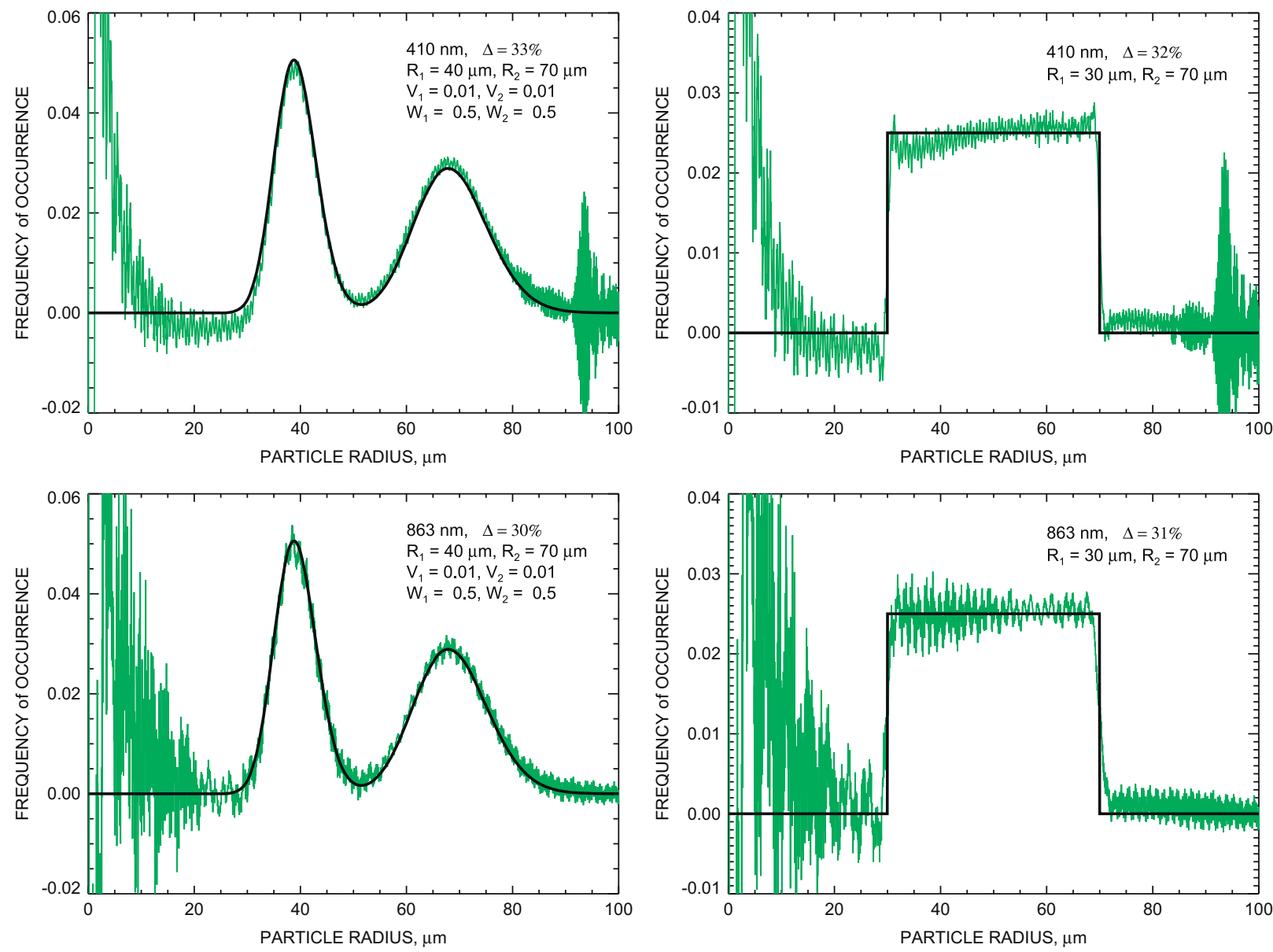

Fig. 4. Examples of RFT loop-transforms (green) of two model area size distributions (plotted in black for comparison). Left: bimodal gamma distribution with equally (50\% each) weighted modes having 40 and $70 \mu \mathrm{m}$ effective radius and the same effective variance of 0.01 . Right: rectangular distribution which is constant between particle radii of 30 and $70 \mu \mathrm{m}$, and zero otherwise. The plots correspond to 410 (top) and $863 \mathrm{~nm}$ (bottom) wavelengths. (For interpretation of the references to color in this figure legend, the reader is referred to the web version of this article.)

constant contribution to $n_{a}^{\prime}(r)$ can easily be determined from its value at very large $r$ and subtracted. Thus, it is sufficient for us to determine all the $r$-dependent artifact functions up to a constant (even if it is distributiondependent), so we will use the terms "distribution-independent" and "universal" neglecting such additive constant dependence. Our numerical tests confirm that the correction function $\eta(r)$ is mostly universal, i.e., its shape only weakly depends on the particular area distribution $n_{a}(r)$. Thus, as the first step of the correction procedure we compute $\eta(r)$ using a default size distribution $n_{d}(r)$. It is convenient for this purpose to use the constant size distribution $n_{d}(r)=1 / r_{\max }$, where $r_{\max }=100 \mu \mathrm{m}$ is the upper limit of particle radius in our Mie theory dataset $\left(n_{d}\left(r>r_{\max }\right)=0\right.$ is assumed $)$. Thus, we define

$\eta_{d}(r)=n_{d}^{\prime}(r)$

i.e., the result of the loop-RFT applied to $n_{d}(r)$ (subtraction of the first term from Eq. (41) is not necessary, since it is constant). Plots of the resulting correction functions are shown in Fig. 5 (left) for 410 and $863 \mathrm{~nm}$ wavelengths (these functions are (arbitrarily) normalized to satisfy $\left.\eta_{d}\left(r_{\max }\right)=0\right)$. The first step of our correction procedure is simply the subtraction of the pre-computed function $\eta_{d}(r)$ from the initial loop-transform result $n_{a}^{\prime}(r)$. The results of this operation (complemented by a movingwindow smoothing) applied to the distributions from Fig. 4 are shown in Fig. 6 demonstrating improvement in retrieval accuracy from $\Delta \sim 30 \%$ to $5-6 \%$. Analytically these results can be represented in the form

$$
\begin{aligned}
n_{a}^{\prime \prime}(r) & =n_{a}^{\prime}(r)-\eta_{d}(r) \\
& =c^{2} n_{a}(r)+\Delta \eta_{s}(r)+\Delta \eta_{g}(r)
\end{aligned}
$$

(where we omit constant terms), since the universal $\eta_{u}(r)$ cancels from the difference. The oscillatory artifacts in Fig. 6 are consistent with the shape of $\Delta \eta_{s}(r)$, which is a linear combination of $s_{0}(r)$ and $s_{1}(r)$, both exhibiting rapid oscillations with a frequency $\sim \gamma_{\max }$, as can be seen in Fig. 5 (right). Note that these oscillatory patterns have a different shape than the artifacts caused by the truncation of the integration range (ringing effects, Gibbs ripples), whose amplitude decreases with distance from the distribution maximum. In order to remove these artifacts, we 

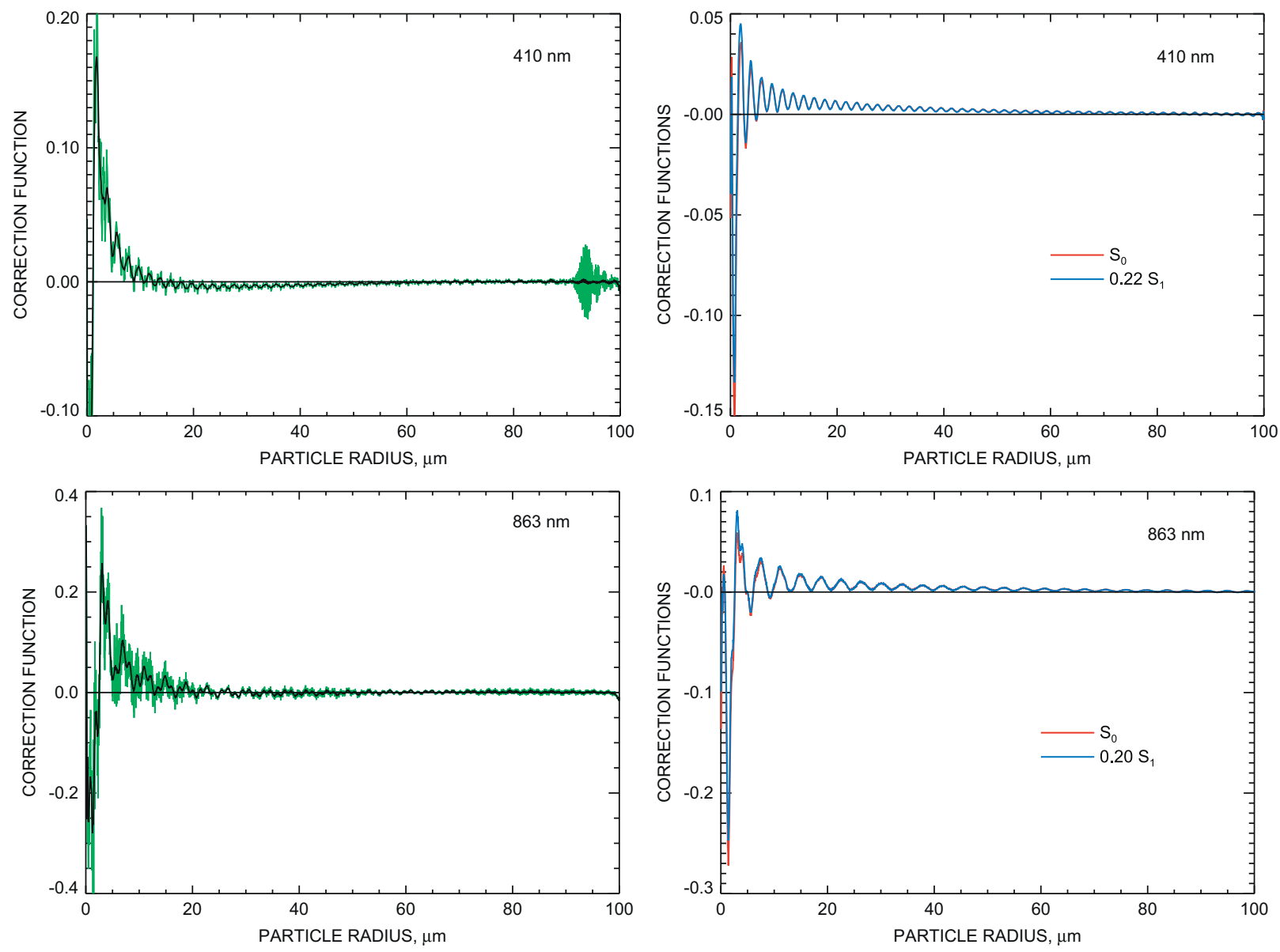

Fig. 5. Plots of the universal correction functions for $410 \mathrm{~nm}$ (top) and $863 \mathrm{~nm}$ (bottom) wavelengths. Left: $\eta_{d}(r)$ (green), black curves depict moving averages to better show the oscillating structure. Right: $s_{0}(r)$ (red) and $s_{1}(r)$, scaled to magnitude of $s_{0}$ (blue). (For interpretation of the references to color in this figure legend, the reader is referred to the web version of this article.)

numerically compute the functions $s_{0}(r)$ and $s_{1}(r)$ according to Eqs. (44) and (45), and then perform a multivariate linear regression on $n_{a}^{\prime \prime}(r)$, as the second step of our cleanup procedure. This regression also includes an exponential function $\exp (-0.07 r)$ that improves the retrieved distribution shape at small $r$ (probably, compensating for the unknown $\eta_{g}(r)$ ), and uses weighting function $r^{-5 / 2}$ (both are empirical guesses). Note that while $s_{0}(r)$ and $s_{1}(r)$ look almost identical (after rescaling) in Fig. 5 (right), they have enough differences in the small particle size range, so both of them should be used in the regression. The resulting size distribution is the residue of this regression. The examples presented in Fig. 7 demonstrate a successful removal of the oscillating artifacts present in Fig. 6 and further accuracy improvement to $\Delta \sim 3-4 \%$. The remaining small differences with the initial size distributions can be attributed to the unknown smooth component $\eta_{g}(r)$, which cannot be objectively separated from the distribution shape.

Note that since $\eta_{d}(r)$ exhibits some slope, the final adjustment of the lower integration limit $\theta_{0}$ should be made based on the condition that the retrieved size distribution has a constant bottom-line after $\eta_{d}$ is subtracted.

\section{Retrievals in the presence of multiple scattering}

While polarized reflectance emerging from a cloud is largely dominated by single scattering by cloud droplets, it also includes a residual contribution from multiple scattering and other factors (Rayleigh scattering, aerosol extinction, etc.). This contribution can be well approximated by a term linear in scattering angle plus a constant $[3,4,1]$, as shown in Eq. (2). The additional term

$\hat{s}(\gamma)=B \gamma+C$

in $\hat{n}_{a}(\gamma)$ generates contamination of $n_{a}^{\prime}(r)$ having the form $s(r)=B s_{1}(r)+C s_{0}(r)$,

where $s_{0}(r)$ and $s_{1}(r)$ are defined by Eqs. (44) and (45). The effect of such contamination generated by $\hat{s}(\gamma)=0.1 \gamma+0.2$ on the retrievals from Fig. 6 is shown in Fig. 8. Obviously, this contamination has the same shape as $\eta_{s}(r)$ described in the previous section (Eq. (43)), and is therefore removed during the second step of our correction procedure leading to the same final results presented in Fig. 7. Thus, this term alone does not present an additional challenge to the retrieval method. However, the retrievals 

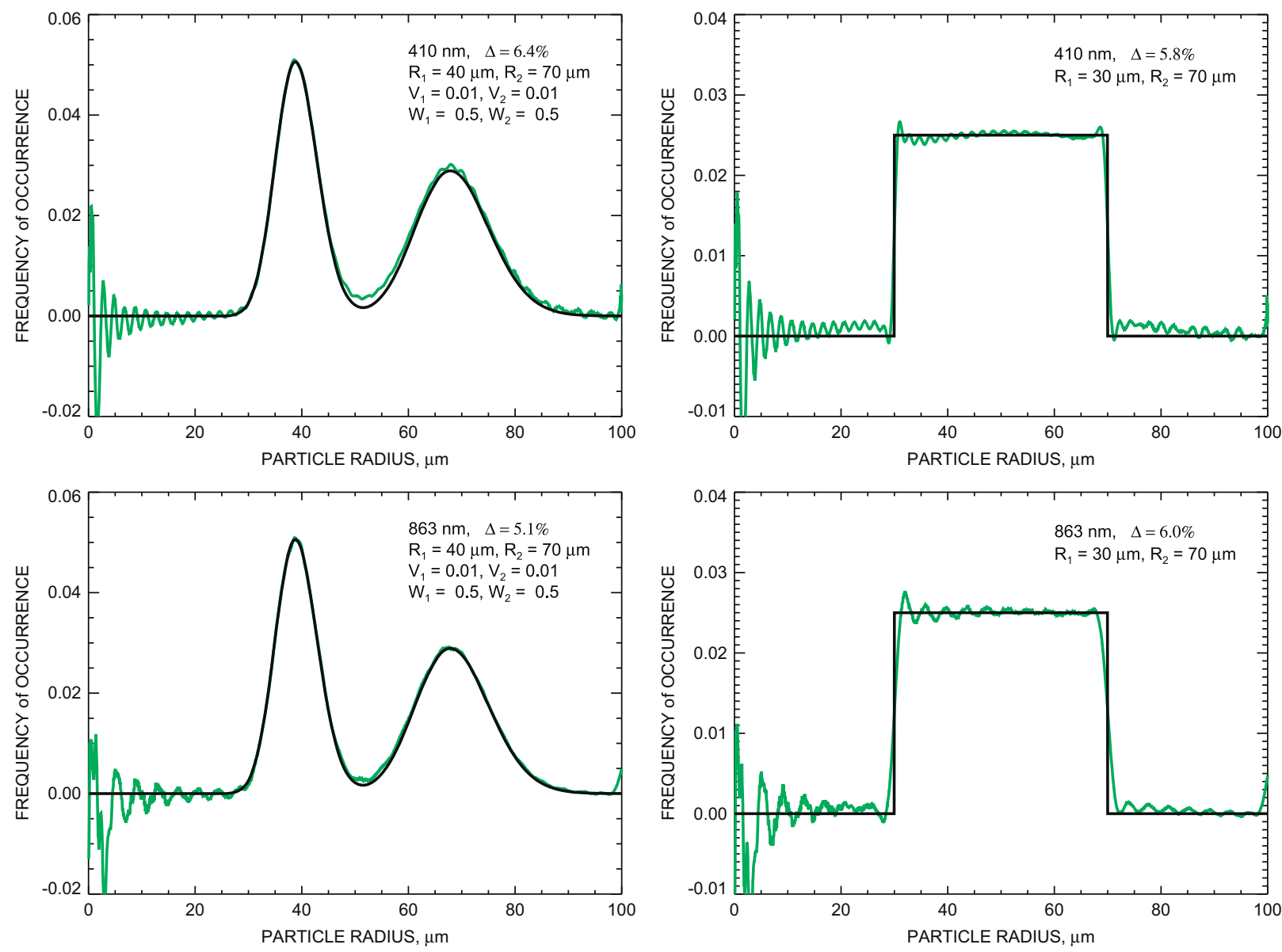

Fig. 6. Same as in Fig. 4, but with the corresponding correction functions $\eta_{d}(r)$ [Fig. 5 (left)] subtracted. In addition to that a moving (11 points $=0.5$ nm) window smoothing was applied. The plots correspond to 410 (top) and $863 \mathrm{~nm}$ (bottom) wavelengths.

from the polarized reflectances having the form of Eq. (2) are complicated by the presence of the unknown coefficient $A$. Indeed, while $\hat{n}_{a}(\gamma)$ is now scaled, the default loop-RFT result $\eta_{d}(r)$ should be scaled accordingly before the subtraction. This suggests that we should combine the first and second steps of the correction method described above into a single multivariate regression in three components: $\eta_{d}(r), s_{0}(r)$, and $s_{1}(r)$ (with a possible additional smooth component). Generally speaking, an increase in the number of regression components could make it less stable (due to increasing possibility of tradeoffs between these components). However, in our case the results of the combined regression correction procedure are practically indistinguishable from those of the original method.

In order to test the RFT-based retrieval algorithm on more realistic data, we applied it to the polarized reflectances simulated by the vector doubling/adding code [20], which has some modifications [21] compared to the original version [16]. The forward modeling of these polarized reflectances (shown in Fig. 1) was performed for thick (optical depth 5) plane-parallel clouds with realistic droplet size distributions. It is clear from Fig. 1 that larger droplets induce more rapid oscillations in polarized reflectance, while increase in $v_{\text {eff }}$ leads to smoothening of the periodic features. The results of inverse RFT with the regression correction procedure applied to these polarized reflectances are shown in Fig. 9. The simulations were done for gamma size distributions with effective radii $r_{\text {eff }}=7.5$ and $17.5 \mu \mathrm{m}$ (right), and effective variances $v_{\text {eff }}=0.01,0.1$, and 0.2 . These numbers are parameters of number size distributions, while the corresponding area size distributions are plotted. The effective radius $r_{\text {eff }}^{\prime}$ and variance $v_{\text {eff }}^{\prime}$ of the area size distribution can be expressed in terms of their number size distribution counterparts as

$v_{\text {eff }}^{\prime}=\frac{v_{\text {eff }}}{1+2 v_{\text {eff }}} \quad$ and $\quad r_{\text {eff }}^{\prime}=r_{\text {eff }}\left(1+2 v_{\text {eff }}\right)$.

The plots show quite good agreement between the retrievals and the initial droplet size distributions used in forward modeling, while some artifacts remain. They include unphysical oscillations in the case of narrow $\left(v_{\text {eff }}=0.01\right)$ distributions, and some shifts towards smaller sizes when $r_{\text {eff }}=17.5 \mu \mathrm{m}\left(0.15 \mu \mathrm{m}\right.$ shift at $v_{\text {eff }}=0.01$, $0.5 \mu \mathrm{m}$ - at $v_{\text {eff }}=0.1$, and $0.75 \mu \mathrm{m}-$ at $\left.v_{\text {eff }}=0.2\right)$. The latter shift, however, may be an artifact of the RT model, rather than of the retrieval method. The retrieval accuracy 

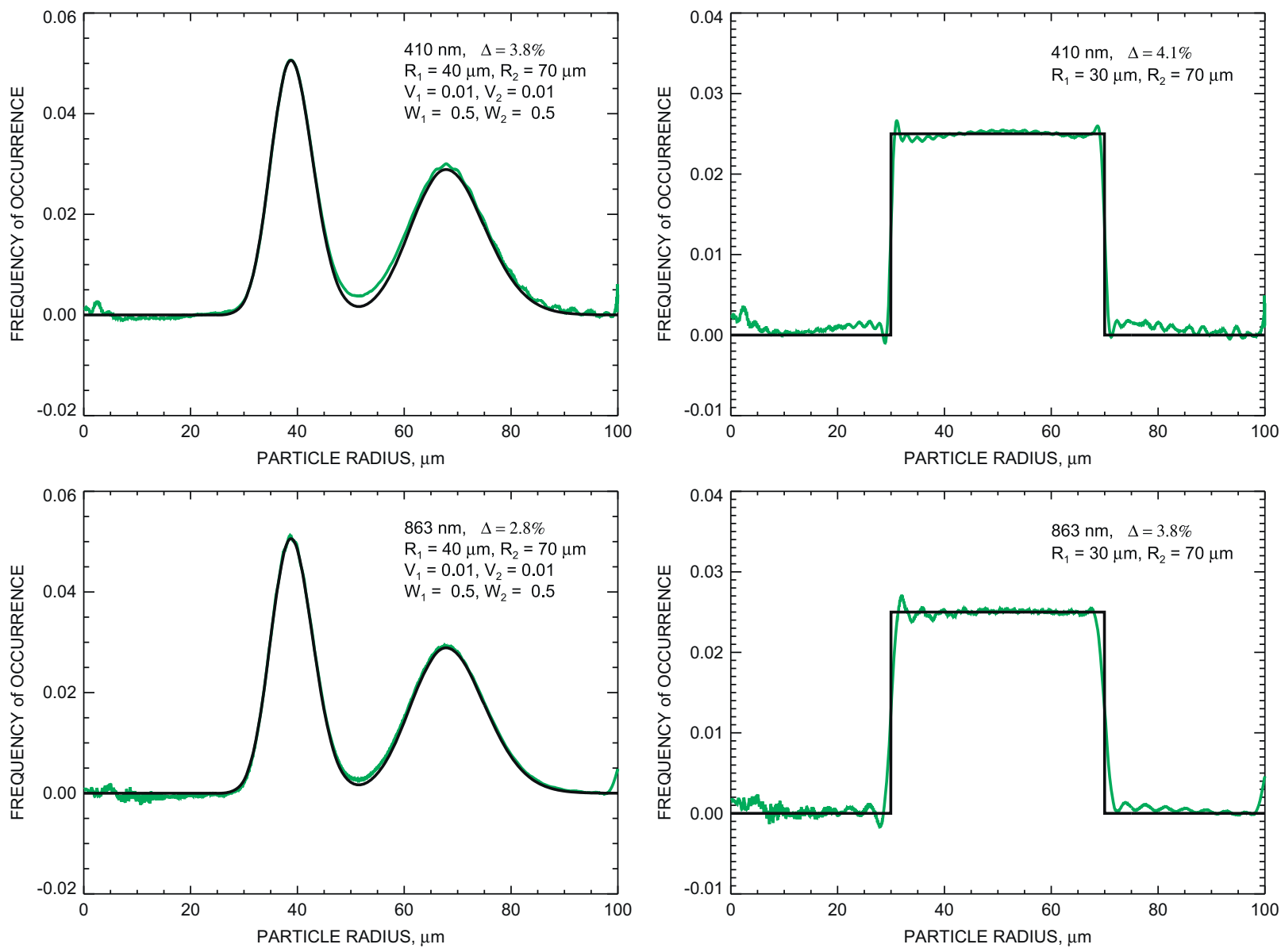

Fig. 7. Same as in Fig. 6, but after application of multivariate regression in $s_{0}(r)$ and $s_{1}(r)$ from Fig. 5 (right), which removes the oscillating artifacts.

appears to be better for larger values of $r_{\text {eff }}$ and/or $v_{\text {eff }}$, since the RFT artifacts are stronger in the small-radius range. In the worst considered case of $r_{\mathrm{eff}}=7.5 \mu \mathrm{m}$ and $v_{\text {eff }}=0.01$, the error is particularly large $(\Delta=50 \%)$, while for $v_{\text {eff }} \geq 0.1$ it is substantially smaller ( $\Delta \leq 14 \%$ ). In the case of $r_{\text {eff }}=17.5 \mu \mathrm{m}$, the accuracy is even better $(\Delta \sim 6-10 \%)$.

\section{Practical application issues}

In this paper we focus on the introduction of a new retrieval algorithm and description of its mathematical basis. Thus, we will only briefly address the issues of application of RFT to real data (such as measurements by the RSP), leaving detailed sensitivity studies to future publications. Some of these sensitivities, which are common to all rainbow-based retrieval techniques are described in detail in another article [1]. They include the effects of rotation to the principal plane, uncertainties in aircraft attitude (pitch and crab angles), multiple scattering contribution (including 3D effects), and the presence of aerosol layer above clouds.

The issues specific to RFT are related to angular range and resolution requirements for the measurements, as well as the information content of the retrieved droplet distribution shape. Our preliminary tests on simulated and real RSP data showed that availability of measurements in full rainbow scattering angle range is essential for applicability of RFT. For example, reduction of the upper limit of this range from $165^{\circ}$ to $155^{\circ}$ significantly impairs the retrievals. This is understandable, since such reduction affects orthogonality of the RFT's basis functions. Degradation of measurement resolution is expected to primarily affect high-frequency components of the angular spectrum, which correspond, as we can see from Fig. 1, to large particles and/or narrow size distributions (as well as to sharp features in the size distribution shape). A detailed quantitative study of these effects is yet to be done. The results of our preliminary tests show that the effect of a factor of four resolution reduction (from the model's $0.2^{\circ}$ to the RSP's $0.8^{\circ}$ ) is practically indistinguishable for area size distributions having monomodal gamma shape with $r_{\text {eff }}$ up to $70 \mu \mathrm{m}$ (it causes only a few per cent increase in $\Delta$ ). RFT with $1.6^{\circ}$ resolution (factor of 8 degradation) works fine for relatively wide distributions $\left(v_{\text {eff }}=0.1-0.2\right)$, while in the case of a narrow distribution $\left(v_{\text {eff }}=0.01\right)$ its performance worsens. However, even in the latter case it still can adequately resolve the distribution maximum for $r_{\text {eff }}$ values up to $40 \mu \mathrm{m}$ (but with increase in $\Delta$ from $7 \%$ to $35 \%$ ). These observations suggest that RFT should not be expected to perform well on datasets with angular resolution worse than 

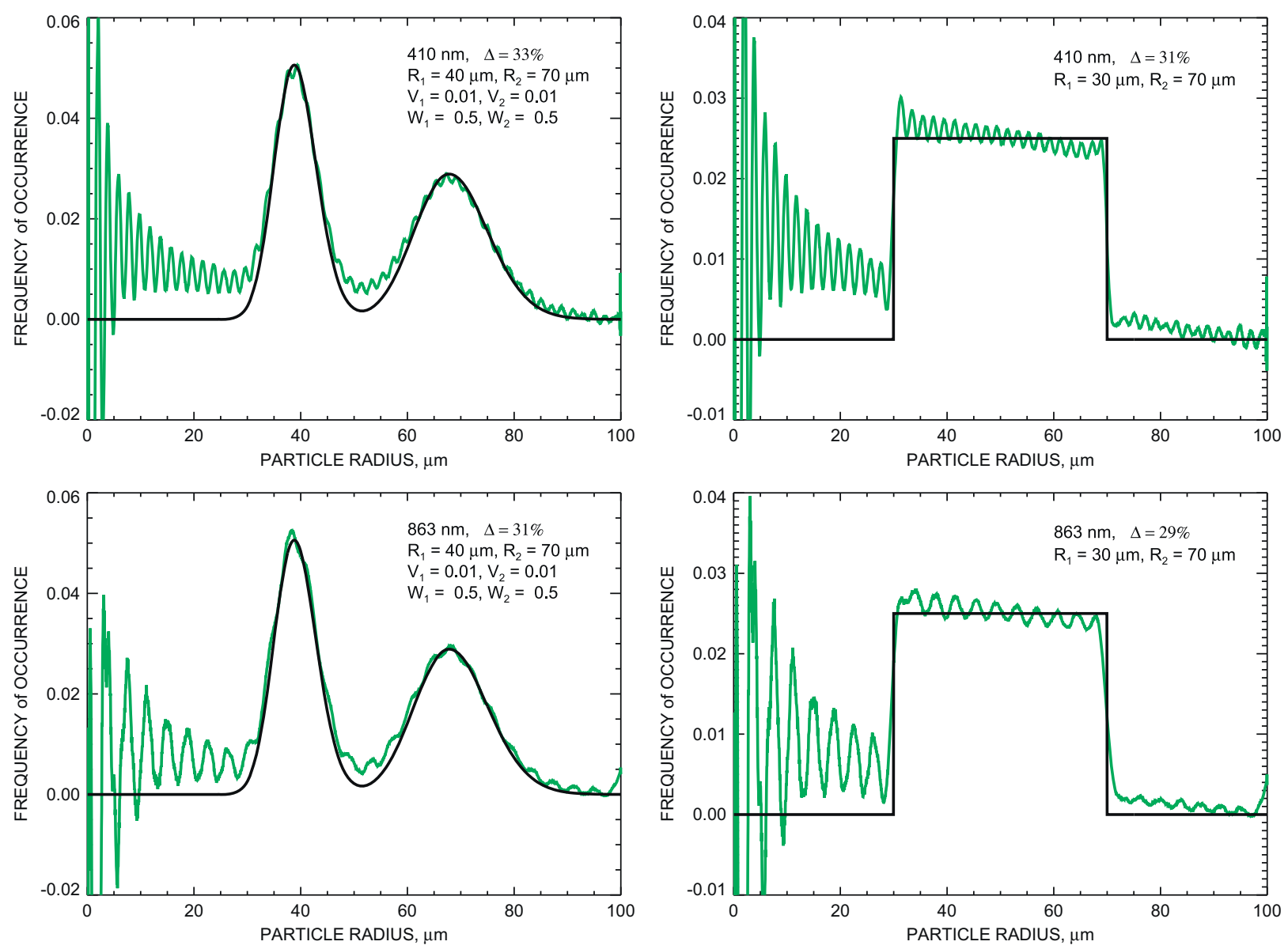

Fig. 8. Same as in Fig. 6, but with the effects of contamination of $\hat{n}_{a}(\gamma)$ with $s(\gamma)=0.1 \gamma+0.2$.

$2^{\circ}$, since the droplet size distributions at the cloud top (which dominate the airborne polarimetric measurements) are typically narrow.

Unlike fitting techniques, integral transforms (including the RFT) cannot be forced to constrain their results to a certain family of functions, such as statistical distributions (i.e., positive normalized functions). This means that while RFT is expected to adequately determine the functional shape of a droplet size distribution, presence of noise and negative values may impact the integration of this distribution over the size range, leading to inaccurate computation of its norm and moments. In the case of a generic distribution shape, this problem needs further study. However, when the size distribution is expected to have gamma distribution shape, its effective radius and variance can be derived without integration. Fortunately, this is the case for the droplet size distributions at the cloud top, which are important for airborne polarimetric measurements. Their tendency to have gamma distribution shape was confirmed by both theoretical model [22,23], and airborne in situ measurements (cf. [24]). Our own results of application of RFT to real RSP data also support this assertion. While a direct functional fitting can be used in order to derive the distribution parameters in this case, we propose another, computationally simpler, approach based on variation of the distribution in the vicinity of its maximum (which is usually free from RFT-generated noise). This approach uses three metrics of the retrieved distribution shape $n(r)$ : position of the distribution maximum (mode radius)

$r_{\max }=r_{\mathrm{eff}}\left(1-3 v_{\mathrm{eff}}\right)$

and the two ratios

$\rho=\frac{r}{r_{\max }} \quad$ and $\quad R=\frac{n(r)}{n\left(r_{\max }\right)}$.

Note that none of these metrics requires proper normalization of the retrieved distribution. Then, we derive $v_{\text {eff }}$, first, from the relation

$\ln R=\left[\frac{1}{v_{\text {eff }}}-3\right][\ln \rho+(1-\rho)]$

for some fixed value of $\rho$ and the corresponding value of $R$ (we found $\rho=0.8$ to be optimal for $r>r_{\max }$ ). After that we determine $r_{\text {eff }}$ from Eq. (51). This method produces good results, when applied to the distributions from Fig. 9, especially for $v_{\text {eff }}=0.01$, when the errors in $r_{\text {eff }}$ and $v_{\text {eff }}$ are less than $0.1 \mu \mathrm{m}$ and 0.01 , respectively. For $v_{\text {eff }}=0.1$ and 0.2 , the corresponding errors are larger, but they are still less than $0.5 \mu \mathrm{m}$ in $r_{\text {eff }}$ and 0.1 in $v_{\text {eff }}$. 

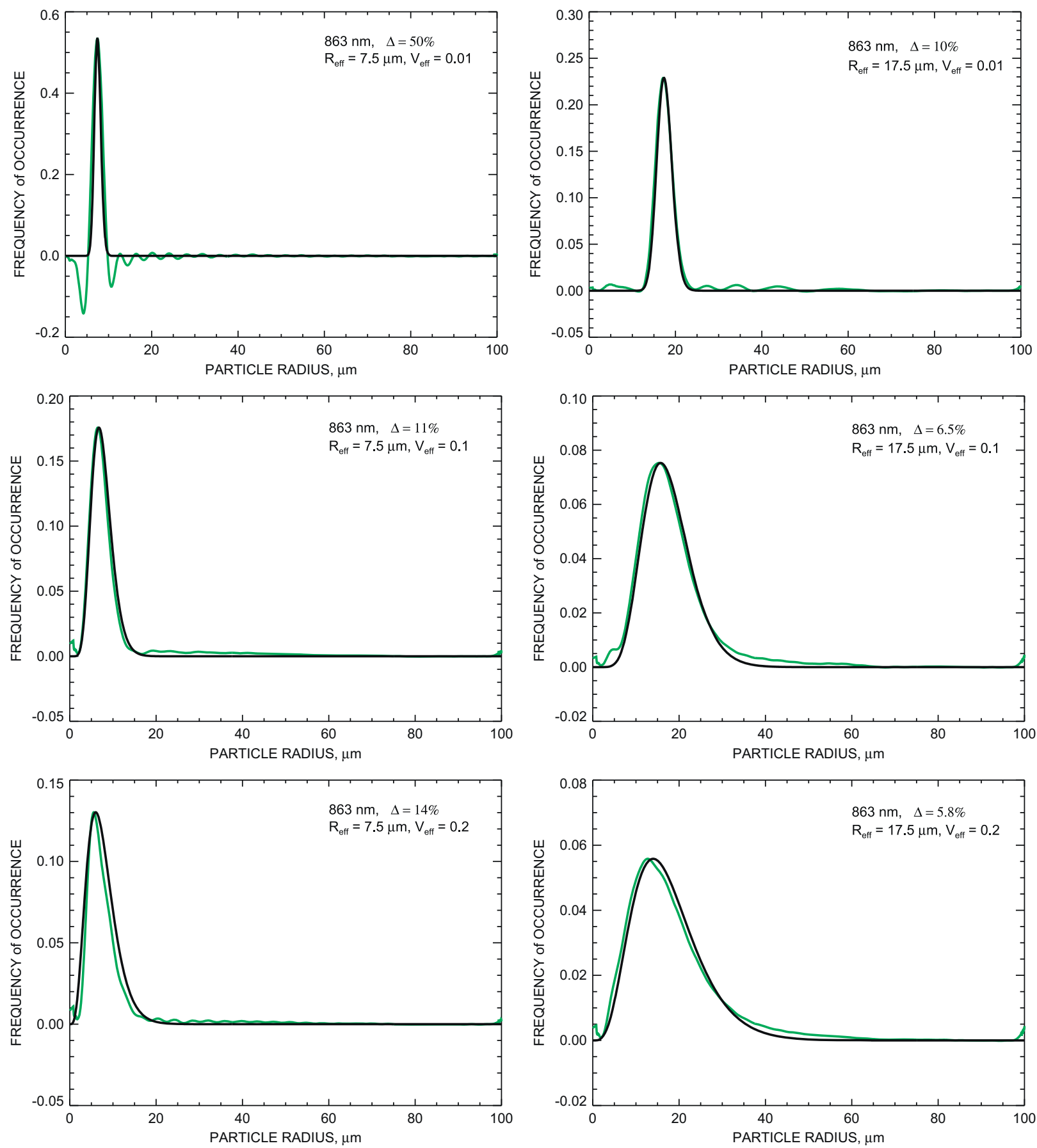

Fig. 9. Results of the inverse RFT (with regression correction) applied to polarized reflectances simulated using a vector radiative transfer code (accounting for multiple scattering contribution). The simulations were made for gamma size distributions with $r_{\text {eff }}=7.5$ (left) and $17.5 \mu$ m (right), and $v_{\text {eff }}=0.01,0.1$, and 0.2 (from top to bottom). Note that these numbers are parameters of the number size distributions, while the corresponding area size distributions are plotted: the retrieved (green) and the original (black). (For interpretation of the references to color in this figure legend, the reader is referred to the web version of this article.)

\section{Conclusions}

We described a new approach (the rainbow Fourier transform) to the retrieval of cloud droplet size distributions from polarized reflectances in the rainbow scattering angle range $\left(135^{\circ}-165^{\circ}\right)$, where they are dominated by single scattering. This method is based on the observation that these polarized reflectances computed using the Mie theory for a range of (monodisperse) particle radii as functions of reduced scattering angle $\gamma$ form a proxy basis of an integral transform (similar to the sine Fourier transform or the Bessel transform on the positive 
semi-axis). The direct transform applied to a given droplet size distribution coincides with the computation of the corresponding polarized reflectance. The inverse transform applied to this polarized reflectance as a function of scattering angle yields a proxy of the original size distribution. To obtain the distribution itself, a simple regression-based correction procedure is applied, which removes retrieval artifacts from this proxy function. Our analytical modeling suggested that these artifacts are caused by the lack of orthogonality of the RFT's base functions. We demonstrated good performance of the described technique using various sample droplet size distributions (bimodal gamma and rectangular) at 410 and $863 \mathrm{~nm}$ wavelengths marking the boundaries of the visible spectral range. We also showed using RT simulations that our approach works well in realistic situations, when the measurements are made in the presence of multiple scattering.

The main advantage of the RFT-based retrieval algorithm compared to the currently used fitting methods $[3,4,1]$ is that this technique is non-parametric, i.e., it does not require any a priori knowledge of the droplet size distribution functional shape (including the number of modes). This also makes our algorithm computationally fast, since its computations used for inversions are essentially the same as those used for the forward modeling. There is no fitting using look-up tables.

We expect that the method described has a potential to detect drizzle in clouds along with the cloud droplet distribution. However, successful use of the RFT-based technique imposes certain requirements on the measurements, including high angular resolution (better than $2^{\circ}$ ), wide angular range (close to the full rainbow range, which can be observed in solar principle plane measurements), and high measurement accuracy (low instrumental noise). This method will be applied to the RSP data from a number of field campaigns.

\section{Acknowledgments}

This basic research was funded by the NASA Radiation Sciences Program managed by Hal Maring and by the NASA Glory Mission project.

\section{Appendix A. Effects of weak orthogonality}

Let us assume that the lack of orthogonality of the RFT basis functions $F(r, \gamma)$ is caused by an additive term $g(r, \gamma)$, i.e., these functions can be represented in the form

$F(r, \gamma)=c H(r, \gamma)+g(r, \gamma)$,

where $c$ is a constant, and $H(r, \gamma)$ satisfy the orthogonality conditions (16) and (17) with the weights from Eq. (35). $H(r, \gamma)$ can be used as the kernel of another integral transform

$\hat{n}_{H}(\gamma)=\int_{0}^{\infty} n_{a}(r) H(r, \gamma) \mathrm{d} r$ which, unlike RFT, is exact, i.e.

$n_{a}(r)=\int_{0}^{\gamma_{\max }} \hat{n}_{H}(\gamma) H(r, \gamma) \gamma^{2} \mathrm{~d} \gamma$

Here, we neglect the effect of finiteness of the integration limit $\gamma_{\max }$, which generally may cause artificial oscillations (ringing artifacts, Gibbs ripples), since the latter do not show up in numerical tests. The result of the original RFT (F-transform, Eq. (36)) can be expressed in terms of this $H$-transform as

$\hat{n}_{F}(\gamma)=c \hat{n}_{H}(\gamma)+\bar{g}(\gamma)$,

where

$\bar{g}(\gamma)=\int_{0}^{\infty} n_{a}(r) g(r, \gamma) \mathrm{d} r$.

Then, the result of the inverse RFT (Eq. (37)) can be written as

$$
\begin{aligned}
n_{a}^{\prime}(r) & =\int_{0}^{\gamma_{\max }}\left[c \hat{n}_{H}(\gamma)+\bar{g}(\gamma)\right] F(r, \gamma) \gamma^{2} \mathrm{~d} \gamma \\
& =c^{2} n_{a}(r)+\eta(r),
\end{aligned}
$$

where

$\eta(r)=\eta_{0}(r)+\eta_{1}(r)$

with

$\eta_{0}(r)=c \int_{0}^{\gamma_{\max }} \hat{n}_{H}(\gamma) g(r, \gamma) \gamma^{2} \mathrm{~d} \gamma$

and

$\eta_{1}(r)=\int_{0}^{\gamma_{\max }} \bar{g}(\gamma) F(r, \gamma) \gamma^{2} \mathrm{~d} \gamma$

describe the non-orthogonality artifacts in the loop RFT. Using Eq. (A.2) and changing the integration order (we assume all the convergence properties necessary for this), Eq. (A.8) can be written as

$$
\begin{aligned}
\eta_{0}(r) & =c \int_{0}^{\gamma_{\max }} \gamma^{2} \mathrm{~d} \gamma g(r, \gamma) \int_{0}^{\infty} \mathrm{d} r^{\prime} n_{a}\left(r^{\prime}\right) H\left(r^{\prime}, \gamma\right) \\
& =\int_{0}^{\infty} n_{a}\left(r^{\prime}\right) h\left(r, r^{\prime}\right) \mathrm{d} r^{\prime},
\end{aligned}
$$

where

$h\left(r, r^{\prime}\right)=c \int_{0}^{\gamma_{\max }} g(r, \gamma) H\left(r^{\prime}, \gamma\right) \gamma^{2} \mathrm{~d} \gamma$

Similarly, Eq. (A.9) can be written in the following form, using Eq. (A.5):

$$
\begin{aligned}
\eta_{1}(r) & =c \int_{0}^{\gamma_{\max }} \gamma^{2} \mathrm{~d} \gamma F(r, \gamma) \int_{0}^{\infty} \mathrm{d} r^{\prime} n_{a}\left(r^{\prime}\right) g\left(r^{\prime}, \gamma\right) \\
& =\int_{0}^{\infty} n_{a}\left(r^{\prime}\right) f\left(r^{\prime}, r\right) \mathrm{d} r^{\prime},
\end{aligned}
$$

where

$f\left(r, r^{\prime}\right)=\int_{0}^{\gamma_{\max }} g(r, \gamma) F\left(r^{\prime}, \gamma\right) \gamma^{2} \mathrm{~d} \gamma$.

The function $h\left(r, r^{\prime}\right)$ in Eq. (A.10) can be expressed in terms of $f\left(r, r^{\prime}\right)$ as

$h\left(r, r^{\prime}\right)=f\left(r, r^{\prime}\right)-y\left(r, r^{\prime}\right)$, 
where

$y\left(r, r^{\prime}\right)=\int_{0}^{\gamma_{\max }} g(r, \gamma) g\left(r^{\prime}, \gamma\right) \gamma^{2} \mathrm{~d} \gamma$

(obviously, $y$ is symmetric: $y\left(r, r^{\prime}\right)=y\left(r^{\prime}, r\right)$ ). In this notation the expression for $\eta(r)$ has the following form:

$\eta(r)=\int_{0}^{\infty} n_{a}\left(r^{\prime}\right) p\left(r, r^{\prime}\right) \mathrm{d} r^{\prime}$

where

$p\left(r, r^{\prime}\right)=f\left(r, r^{\prime}\right)+f\left(r^{\prime}, r\right)-y\left(r, r^{\prime}\right)$

is symmetric function: $p\left(r, r^{\prime}\right)=p\left(r^{\prime}, r\right)$.

Our numerical tests show that the shape of $\eta(r)$ is almost independent of the size distribution $n_{a}(r)$ (it may, however, include a distribution-dependent constant, which can always be removed by taking into account that $n_{a}(r)=0$ at very large $r$. As we show below, complete distribution-independence in the above sense is achieved if the additive term $g(r, \gamma)$ does not depend on particle radius: $g=g_{0}(\gamma)$. This allows us to consider the $r$-dependent part of the term $g$ as a small perturbation

$g(r, \gamma)=g_{0}(\gamma)+\varepsilon g_{1}(r, \gamma)$

where $\varepsilon$ is a small parameter. In this case

$f\left(r, r^{\prime}\right)=f_{0}\left(r^{\prime}\right)+\varepsilon f_{1}\left(r, r^{\prime}\right)$,

where

$f_{0}\left(r^{\prime}\right)=\int_{0}^{\gamma_{\max }} g_{0}(\gamma) F\left(r^{\prime}, \gamma\right) \gamma^{2} \mathrm{~d} \gamma$

and

$f_{1}\left(r, r^{\prime}\right)=\int_{0}^{\gamma_{\max }} g_{1}(r, \gamma) F\left(r^{\prime}, \gamma\right) \gamma^{2} \mathrm{~d} \gamma$.

In computation of $y\left(r, r^{\prime}\right)$, we omit terms quadratic in $\varepsilon$

$y\left(r, r^{\prime}\right)=y_{0}+\varepsilon\left[y_{1}(r)+y_{1}\left(r^{\prime}\right)\right]$,

where

$y_{0}=\int_{0}^{\gamma_{\max }} g_{0}^{2}(\gamma) \gamma^{2} \mathrm{~d} \gamma$

is a constant, and

$y_{1}(r)=\int_{0}^{\gamma_{\max }} g_{0}(\gamma) g_{1}(r, \gamma) \gamma^{2} \mathrm{~d} \gamma$.

Thus

$p\left(r, r^{\prime}\right)=f_{0}(r)+f_{0}\left(r^{\prime}\right)+\varepsilon\left[f_{1}\left(r, r^{\prime}\right)+f_{1}\left(r^{\prime}, r\right)\right]-y_{0}-\varepsilon\left[y_{1}(r)+y_{1}\left(r^{\prime}\right)\right]$

and

$\eta(r)=\eta_{u}(r)+\eta_{s}(r)+\eta_{g}(r)+C_{\eta}$,

where

$\eta_{u}(r)=f_{0}(r)-\varepsilon y_{1}(r)$

is a distribution-independent (universal) function

$C_{\eta}=\bar{f}_{0}-y_{0}-\varepsilon \bar{y}_{1}$

is a constant (here the bar denotes an average with $n_{a}\left(r^{\prime}\right)$ ), while the terms

$\eta_{s}(r)=\varepsilon \int_{0}^{\infty} n_{a}\left(r^{\prime}\right) f_{1}\left(r^{\prime}, r\right) \mathrm{d} r^{\prime}$ and

$\eta_{g}(r)=\varepsilon \int_{0}^{\infty} n_{a}\left(r^{\prime}\right) f_{1}\left(r, r^{\prime}\right) \mathrm{d} r^{\prime}$

are the functions of particle radius, which depend on the size distribution shape. Note that these terms disappear when $\varepsilon=0$ (i.e., when $g$ depends on $\gamma$ only), and $\eta(r)$ becomes universal (up to a constant). To understand the structure of $\eta_{s}(r)$ and $\eta_{g}(r)$, let us represent $g_{1}(r, \gamma)$ by its Taylor's series expansion in $\gamma$

$g_{1}(r, \gamma)=\sum_{0}^{\infty} g_{1 i}(r) \gamma^{i}$

which we assume to be uniformly convergent on the finite interval $\left[0, \gamma_{\max }\right]$ for all $r$. Then

$f_{1}\left(r, r^{\prime}\right)=\sum_{0}^{\infty} g_{1 i}(r) s_{i}\left(r^{\prime}\right)$

where

$s_{i}(r)=\int_{0}^{\gamma_{\max }} \gamma^{i} F(r, \gamma) \gamma^{2} \mathrm{~d} \gamma$

Thus

$\eta_{s}(r)=\varepsilon \sum_{0}^{\infty} \bar{g}_{1 i} s_{i}(r)$

and

$\eta_{g}(r)=\varepsilon \sum_{0}^{\infty} g_{1 i}(r) \overline{S_{i}}$

The functions $s_{i}(r)$ show rapid oscillations with frequency $\sim \gamma_{\max }$. The plots of $s_{0}$ and $s_{1}$ are shown in Fig. 5 (right). On the other hand, $g_{1 i}(r)$ are expected to be smooth slowly varying functions of $r$. This makes it difficult (if even possible) to separate them from the size distribution features. Fortunately, they appear to be small in magnitude. It appears from our numerical simulations that for an adequate fit of $n_{a}^{\prime}(r)$ only first two terms in the expansion of $g_{1}(r, \gamma)$ are needed for an adequate fit of $n_{a}^{\prime}(r):$

$g_{1}(r, \gamma) \approx g_{10}(r)+g_{11}(r) \gamma$.

This simplifies the expression for $\eta_{s}(r)$ :

$\eta_{s}(r) \approx \varepsilon\left[\bar{g}_{10} s_{0}(r)+\bar{g}_{11} s_{1}(r)\right]$.

\section{References}

[1] Alexandrov MD, Cairns B, Emde C, Ackerman AS, van Diedenhoven B. Characterization of cloud droplet size distributions based on polarized reflectance measurements by the research scanning polarimeter: sensitivity study. Atmos Chem Phys; to be submitted.

[2] Mishchenko MI, Travis LD, Lacis AA. Multiple scattering of light by particles: radiative transfer and coherent backscattering. Cambridge University Press; 2006.

[3] Bréon FM, Goloub P. Cloud droplet effective radius from spaceborne polarization measurements. Geophys Res Lett 1998;25:1879-82.

[4] Bréon FM, Doutriaux-Boucher M. A comparison of cloud droplet radii measured from space. IEEE Trans Geosci Remote Sensing 2005;43:1796-805.

[5] Platnick S, Valero FPJ. A validation study of a satellite cloud retrieval during ASTEX. J Atmos Sci 1995;52:2985-3001.

[6] Coddington OM, Pilewskie P, Redemann J, Platnick S, Russell PB, Schmidt KS, et al. Examining the impact of overlying aerosols on 
the retrieval of cloud optical properties from passive remote sensing. J Geophys Res 2010;115:D10211.

[7] Cairns B, Russell EE, Travis LD. Research scanning polarimeter: calibration and ground-based measurements. In: Proceedings of SPIE, vol. 3754; 1999. p. 186-97.

[8] Cairns B, Waquet F, Knobelspiesse K, Chowdhary J, Deuzé JL. Polarimetric remote sensing of aerosols over land surfaces. In: Kokhanovsky AA, Leeuw GD, editors. Satellite aerosol remote sensing over land. Springer; 2009. p. 295-325.

[9] Waquet F, Cairns B, Knobelspiesse K, Chowdhary J, Travis LD, Schmid B, et al. Polarimetric remote sensing of aerosols over land. J Geophys Res 2009;114:D01206.

[10] Chowdhary J, Cairns B, Mishchenko MI, Hobbs PV, Cota GF, Redemann J, et al. Retrieval of aerosol scattering and absorption properties from photopolarimetric observations over the ocean during the CLAMS experiment. J Atmos Sci 2005;62:1093-117.

[11] Knobelspiesse KD, Cairns B, Schmid B, Roman MO, Schaaf CB. Surface BRDF estimation from an aircraft compared to MODIS and ground estimates at the Southern Great Plains site. J Geophys Res 2008;113:D20105.

[12] Knobelspiesse KD, Cairns B, Redemann J, Bergstrom RW, Stohl A. Simultaneous retrieval of aerosol and cloud properties during the MILAGRO field campaign. Atmos Chem Phys 2011;11:6245-63.

[13] Knobelspiesse KD, Cairns B, Ottaviani M, Ferrare R, Hair J, Hostetler C, et al. Combined retrievals of boreal forest fire aerosol properties with a polarimeter and lidar. Atmos Chem Phys 2011;11:7045-67.

[14] Mishchenko MI, Cairns B, Kopp G, Schueler CF, Fafaul BA, Hansen JE, et al. Accurate monitoring of terrestrial aerosols and total solar irradiance: introducing the glory mission. Bull Am Meteorol Soc 2007;88:677-91.

[15] Grandy WT. In: Scattering of waves from large spheres.Cambridge University Press; 2000.

[16] Hansen JE, Travis LD. Light scattering in planetary atmospheres. Space Sci Rev 1974;16:527-610.

[17] Vallée O, Soares M. Airy functions and applications to physics. Imperial College Press; 2004.

[18] Abramowitz M, Stegun IA. Handbook of mathematical functions. Washington: National Bureau of Standards; 1964 [Reprinted by Dover, New York, 1972].

[19] Alexandrov MD, Ackerman AS, Marshak A. Cellular statistical models of broken cloud fields. Part II: comparison with a dynamical model and statistics of diverse ensembles. J Atmos Sci 2010;67: 2152-70.

[20] Cairns B, Carlson BE, Lacis AA, Russell EE. An analysis of groundbased polarimetric sky radiance measurements. In: Proceedings of SPIE, vol. 3121; 1997. p. 382-93.

[21] de Haan JF, Bosma PB, Hovenier JW. The adding method for multiple scattering calculations of polarized light. Astron Astrophys 1987;183:371-2.

[22] Khvorostyanov VI, Curry JA. Toward the theory of stochastic condensation in clouds. Part II: analytical solutions of the gamma-distribution type. J Atmos Sci 1999;56:3997-4013.

[23] Khvorostyanov VI, Curry JA. Analytical solutions to the stochastic kinetic equation for liquid and ice particle size spectra. Part I: small-size fraction. J Atmos Sci 2008;65:2025-43.

[24] Pruppacher HR, Klett JD. Microphysics of clouds and precipitation. Kluwer; 1997. 